\title{
Correlation of Handgrip Strength to Postoperative Outcomes in Rotator Cuff Repair: A Preliminary Report
}

\author{
Y. Karanjkar ${ }^{1}$, A. Prabhu², K. Vishal ${ }^{2}$ \\ 1 Postgraduate student \\ 2 Assistant Professor, Department of Physiotherapy, School of Allied Health Sciences, Manipal Academy of \\ Higher Education, Manipal, Karnataka, India
}

\section{CORRESPONDING AUTHOR:}

\section{Kavitha Vishal}

Department of Physiotherapy

School of Allied Health Sciences

Manipal Academy of Higher Education

Manipal-576104, Karnataka, India.

E-mail: kavitha.vishal@manipal.edu

DOI:

10.32098/mltj.01.2019.15

\begin{abstract}
SUMMARY
Background. Recovery of strength, range of motion and function are considered major determinants of success following rotator cuff repair. The strength of the distal limb measured as handgrip strength is a simple predictor of proximal muscle function. Moreover, grip strength is used to prognosticate outcomes in several conditions. This study investigated whether preoperative handgrip strength can be used as an indicator of outcomes in patients undergoing rotator cuff repair. Methods. A cross sectional study was performed on 21 patients ( 13 males and 8 females; mean age $55.14 \pm 8.88$ years) undergoing rotator cuff repair. Grip strength on the affected side was assessed preoperatively, and correlated with postoperative outcomes measured at fourth month follow up, which included isometric shoulder internal and external rotator strength, active Range of Motion (ROM) of the shoulder, pain assessed using the Numerical Pain Rating Scale (NPRS), and a performance-based shoulder function test, consisting of three activities. Results. Handgrip strength did not correlate with shoulder strength, ROM and pain. However, there was a moderate correlation $(\mathrm{p}=0.505, \mathrm{r}=0.020)$ between pre-operative grip strength and shoulder function. Conclusion. Handgrip strength did not correlate with any of the outcomes of rotator cuff repair. Although a relationship between grip strength and shoulder function was shown, the correlation was moderate, indicating that multiple factors could contribute to the outcomes in individuals undergoing rotator cuff repair.
\end{abstract}

KEY WORDS

hand function; hand gripping; rotator cuff muscles; rotator cuff injury; shoulder function

\section{BACKGROUND}

Rotator cuff are a group of muscles and tendons that surround the shoulder joint, centering the humeral head within the glenoid cavity, and contributing to the stability of the shoulder girdle. Rotator cuff injuries (RCI) constitute a significant cause of shoulder dysfunction, and rank third in its contribution to shoulder pain (1-3). Multiple factors may affect outcome after rotator cuff injury, with both intrinsic and extrinsic factors playing an important role (4). Majority of patients with an RCI undergo Rotator Cuff Repair (RCR), as there is established evidence for surgical repair over conservative management for small and medium rotator cuff tears (5-7). Determinants of a successful RCR are pain reduction, integrity of cuff, strength, ROM, function, and quality of life (8).

Handgrip strength is an indicator of the overall strength of the upper extremity and is correlated well with mortality and morbidity of several conditions $(9,10)$. Researchers have studied the relationship between preoperative muscle weakness as assessed by hand grip strength, and postoperative outcomes. Studies report activation of proximal shoulder muscles in response to distal extremity tasks (11-18). Horsley et al. (2016) found a relationship between handgrip strength and rotator cuff muscle strength in healthy individuals (11). This established relationship was mainly due to the simultaneous activation of the distal and proximal arm muscles and probable mechanics that stabilize the shoulder 
and the elbow, either via force transmission or through the neural circuits $(11,18)$.

Hand grip strength is a simple, reliable and objective method to evaluate the strength of the upper extremity. Since grip strength correlates with rotator cuff function, it can be used to monitor rotator cuff function and limit the evaluation of the shoulder, especially during the early postoperative period. An association between grip strength and rotator cuff activity has been reported in normal shoulders, however, such a relationship in rotator cuff injury has seldom been explored. Furthermore, we wanted to determine whether a pre-operative decrement of grip strength affects postoperative outcomes in RCR. Hence, the objective of this study was to evaluate the relationship between preoperative handgrip strength to post-operative shoulder internal and external rotator strength, pain, ROM, and function in patients undergoing RCR.

\section{MATERIALS AND METHODS}

Patients with degenerative rotator cuff tears undergoing RCR referred to the department of physiotherapy of a tertiary care hospital participated in the study. Study protocol was approved by Institutional Research Committee (IRC), Institutional Ethics Committee (IEC 58/2017) and registered in the Clinical Trial Registry (CTRI/2017/03/008213). All participants gave a written informed consent, and were given a thorough explanation of the study procedures. The study was conducted from January 2016 to December 2017.

Participants were individuals above 18 years of age (range 41-68), who presented with degenerative RCI confirmed by ultrasound scans. Patients who had recent shoulder fractures, labral tears, cervical radiculopathy, trauma to the hand preventing grip strength assessment, and neurological involvement of the upper extremity, were excluded. All participants in the study underwent arthroscopic rotator cuff repair and received routine physiotherapy from the first post-operative day. Patient characteristics like age, gender, height, weight, hand dominance, duration of symptoms, comorbid factors like diabetes mellitus, hypertension, were recorded. Measurement of hand grip strength on the affected side was measured pre-operatively. Postoperative outcomes were recorded at fourth month follow up visit of the participant. Ultrasound scans at third month were evaluated to identify the status of tendon healing.

Grip strength was recorded using Jamar handheld dynamometer in a standardized position (Sammons Preston, Inc., USA). The participant sat on a chair with their back unsupported and the handheld dynamometer on the side to be tested. The handle of the dynamometer was adjusted in the third handle position, with the shoulder to be evaluated in adduction, elbow in flexion, forearm in mid prone and wrist in 15 degrees of extension without any hand rest. The participant's feet were planted on the ground. They were instructed to do a maximal gripping activity. Three trials were taken with the rest period of 15 seconds between each test, and the average of these three trials was chosen as the final score. Impaired grip strength was defined as the inability to achieve 85 percentile of the hand strength of age and gender-matched controls (19).

Post-operative outcome evaluation comprised of shoulder strength using isometric dynamometer $\left(\mathrm{Ch}\right.$ atillon ${ }^{\circledR}$, a registered trademark of AMETEK, Inc., USA). External and internal rotator strength of the rotator cuff was assessed using an isometric hand-held dynamometer. The participant sat on a plinth with his/her unaffected hand stabilizing the distal aspect of the arm of the extremity to be tested to avoid abduction at the shoulder joint.

To assess the external rotator strength, the tester stood next to the participant on the side of the extremity to be tested. The isometric hand-held dynamometer was placed just proximal to the wrist, and the participant was instructed to perform a maximal pain-free external rotation movement with the forearm in mid pronation and maintain the position against resistance applied for five seconds by the tester through the dynamometer, the reading was recorded. After a rest period of 15 seconds, internal rotator strength was assessed. To assess the internal rotator strength, the tester stood to the opposite of the extremity to be tested. The position of the isometric hand-held dynamometer was same as mentioned earlier for assessing the external rotation strength. The participant was asked to perform a maximal pain-free internal rotation movement with the forearm in mid prone and to maintain the position against the resistance applied by the tester through the dynamometer applied for five seconds; the reading was recorded.

Shoulder function was assessed using function-related test which has good intra-tester and inter-tester reliability. The participants were scored in each of these tests as $0,1,2$, 3 , or 4 depending upon how he/she performed the task, where zero is the full completion of the task and four being least completion of the task. The test comprised of three activities, viz. hand touching the neck, hand touching the scapula and hand touching the opposite scapula performed with the participants in standing position. The participants were categorized for function based on the test (20).

Active ROM of the shoulder in all directions were recorded with a universal goniometer according to standard guidelines (21). Numerical Pain Rating Scale (NPRS) was used to capture the participant's pain intensity on a 11-point scale ranging from 0 ("no pain") to 10 ("worst pain imaginable") (22). 


\section{DATA ANALYSIS}

Data analysis was done using SPSS version 20. Demographic characteristics were summarized using descriptive statistics. As the data followed normal distribution, Pearson's correlation coefficient was used to correlate between grip strength and shoulder strength, ROM, and pain. Spearman's correlation coefficient was used to correlate between grip strength and shoulder function. Level of significance was set at $\mathrm{p} \leq 0.05$.

\section{RESULTS}

Twenty-one patients with rotator cuff repair completed the study. Figure 1 shows the flow of participants during the study. Table I depicts the demographic characteristics of the participants. The mean age of participants was $55.41 \pm 8.88$ years, and all were right dominant. The study group consisted of 10 males and 11 females. The mean and standard deviation of grip strength was $21.01 \pm 9.76 \mathrm{kgs}$. All patients had diminished grip strength before the surgery compared to age and gender-matched controls.

Postoperative outcomes and functional scoring are summarized in table II and III, respectively. The mean external and internal rotator strength $5.39 \pm 1.95,6.51 \pm 2.10 \mathrm{~kg}$ respectively, was significantly low on isometric testing of the shoulder. Table III depicts the number of patients who could achieve the individual scores in the functional activities. Most of the patients were able to accomplish the hand to neck and hand to opposite scapula activity at the $4^{\text {th }}$ month follow up.

Table IV shows the correlation between pre-operative grip strength and shoulder strength, pain, ROM, and function. Grip strength did not correlate with external rotator strength $(\mathrm{r}=0.18, \mathrm{p}=0.41), \mathrm{ROM}(\mathrm{r}=0.24, \mathrm{p}=0.29)$ and pain $(r=-0.15, p=0.49)$. However, grip strength was associated with the hand to neck activity $(\mathrm{r}=0.505, \mathrm{p}=0.020)$ of shoulder function test.

\section{DISCUSSION}

This study aimed to identify the relationship between pre-operative hand grip strength with postoperative outcomes in patients undergoing RCR. Previous studies evaluating hand grip strength suggest that reduced grip strength is associated with significant morbidity. However, the results of the present study indicate no correlation between handgrip strength assessments with shoulder rotator strength, pain, and ROM post RCR.

Grip strength has been found to correlate with rotator cuff function in healthy shoulders. However, this relationship

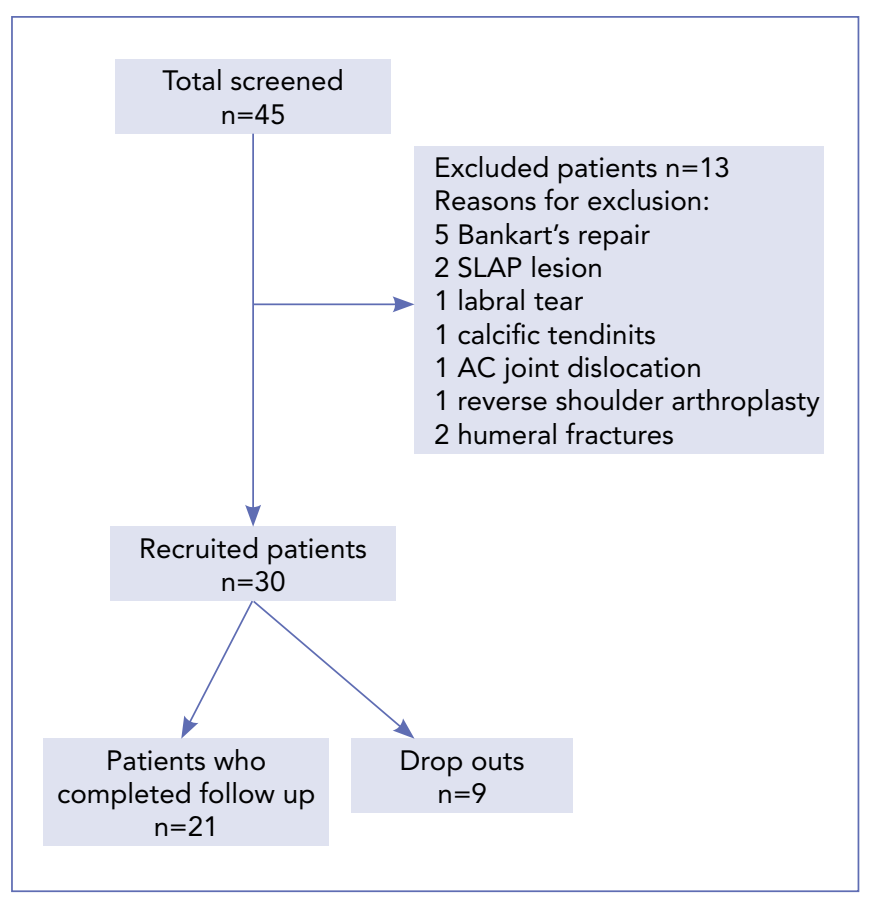

Figure 1 - Flow of Participants.

Table I - Demographic characteristics and grip strength of the participants $(n=21)$.

\begin{tabular}{ll}
\hline Variable & Mean \pm SD (range) \\
\hline Age (years) & $\begin{array}{l}55.41 \pm 8.88 \text { (Range } \\
41-68)\end{array}$ \\
$\begin{array}{l}\text { Preoperative grip strength }(\mathrm{kg}) \\
21.01 \pm 9.76\end{array}$ \\
\hline Variable & $\mathrm{N}$ \\
\hline Gender & 10 \\
male & 11 \\
female & 21 \\
\hline Dominance & 16 \\
right dominant & 05 \\
surgery on dominant extremity & (right) \\
surgery on non-dominant extremity & \\
(left) & \\
\hline Number of tendons repaired & 14 \\
1 tendon & 07 \\
2 tendons & \\
\hline Comorbid factors & 5 \\
diabetes mellitus & 6 \\
\hline hypertension & Median (IQR) \\
\hline Variable & $4(1-183)$ \\
\hline chronicity of pain (weeks) &
\end{tabular}

Kgs, kilograms. 
Table II - Mean and standard deviation of postoperative outcomes of shoulder strength, ROM and pain.

\begin{tabular}{ll}
\hline Variable & Mean (SD) \\
\hline External rotation strength (kgs) & $5.39(1.95)$ \\
\hline Internal rotation strength (kgs) & $6.51(2.10)$ \\
\hline Flexion ROM (degrees) & $129.28(31.03)$ \\
\hline Extension ROM (degrees) & $24.14(9.02)$ \\
\hline Abduction ROM (degrees) & $117.38(36.66)$ \\
\hline Internal rotation ROM (degrees) & $57.71(15.84)$ \\
\hline External rotation ROM (degrees) & $56.19(20.97)$ \\
\hline NPRS & $4.42(1.59)$ \\
\hline
\end{tabular}

Kgs, kilograms; ROM, Range of Motion; NPRS, Numerical Pain Rating Scale.

Table III - Functional scores at fourth month follow up.

\begin{tabular}{lllll}
\hline Functional scoring & 0 & 1 & 2 & 3 \\
\hline Hand to neck $^{1}$ & $6^{4}$ & $12^{4}$ & $1^{4}$ & $2^{4}$ \\
\hline Hand to scapula $^{2}$ & 1 & 7 & 8 & 5 \\
\hline Hand to opposite scapula $^{3}$ & 13 & 7 & 1 & - \\
\hline $\begin{array}{l}1,2,3 \\
\text { represent the number of subjects lying in the functional scores } 0,1,2,3 ;\end{array}$ \\
the classification of function based on the aforementioned scoring system. \\
${ }^{4}$ Shows number of participants.
\end{tabular}

Table IV - Correlation between pre-operative grip strength and shoulder strength, pain, ROM and function.

\begin{tabular}{lll}
\hline Variables & r-value & p-value \\
\hline Shoulder external rotator strength & 0.187 & 0.41 \\
\hline Shoulder internal rotator strength & -0.020 & 0.93 \\
\hline Pain intensity (NPRS) & -0.156 & 0.499 \\
\hline Shoulder flexion ROM & 0.187 & 0.418 \\
\hline Shoulder extension ROM & 0.199 & 0.368 \\
\hline Shoulder abduction ROM & 0.103 & 0.657 \\
\hline Shoulder internal rotation ROM & 0.254 & 0.289 \\
\hline Shoulder external rotation ROM & 0.243 & 0.298 \\
\hline Shoulder function (hand to neck) & $0.505^{1}$ & $0.020^{1}$ \\
\hline Shoulder function (hand to scapula) & -0.101 & 0.665 \\
\hline Shoulder function (hand to opposite scapula) & 0.110 & 0.634 \\
\hline${ }^{1}$ Correlation significant at $\mathrm{p}<0.05$. & &
\end{tabular}

is not necessarily linear in the presence of an injury. Several factors may limit the isometric strength of the external rotators postoperatively. We observed that majority of the participants were apprehensive during isometric testing of the strength, which could have led to poor force produc- tion. A systematic review by Anouk et al., found that postoperative recovery of external rotation strength depends upon several factors like age, gender, tear size, comorbid factors, fatty infiltration and number of tendons repaired $(8,23)$. Moreover, studies have shown that structural integrity post RCR may not translate into satisfactory motor performances of rotator cuff tendons (24).

Tear size and fatty infiltration of tendons have been associated with poorer outcomes postoperatively (24-27). Ultrasound reports of our participants postoperatively revealed tendinopathy of involved as well as adjoining tendons with signs of fatty infiltration and subacromial-subdeltoid bursitis. The preoperative ultrasound reports showed large ( $\mathrm{n}=$ $19)$ and massive $(n=2)$ tears of the rotator cuff tendons for the recruited participants, which may be responsible for the delayed recovery. In our study, these findings could have led to poor outcomes in the participants despite having an adequate grip strength before the surgery.

There was no correlation found between preoperative grip strength and shoulder ROM. One of the factors that possibly could have led to a restricted range was higher pain score $(r=-0.486, p=0.26)$. Participants were given phase wise unsupervised home exercise program, with progression at every month for improving the range and for pain reduction. Adherence to home exercise program postoperatively could have influenced progress. However, this was not monitored in the current study.

The preoperative grip strength and shoulder function (in the activity hand to neck) showed a moderate correlation in spite of having weakness of rotator cuff muscles; this could have been achieved due to compensation from other scapular muscles seen in patients with rotator cuff weakness $(28,29)$. Moreover, further analysis revealed that higher preoperative grip strength yielded better outcomes. There was no significant association between grip strength and other shoulder function test in the activity hand touching the scapula and hand touching the opposite scapula. The possible reason could be due to post-operative shoulder stiffness, as these movements require shoulder to adduct and internally rotate. Post-operative exercises for these patients focused more on elevation and external rotation activities; this could explain the poorer outcomes on other functional tests.

The present study had few limitations. Sample size was small and postoperative outcome assessment at the fourth month is not sufficient to identify the recovery of function in large to massive rotator cuff tears. In spite of having higher impairments of ROM and strength, participants could achieve satisfactory functional levels. This could be attributed to the compensation by other shoulder girdle musculature. Future studies with longer follow up are recommend- 
ed, to confirm whether handgrip strength can be used to prognosticate recovery after surgery in rotator cuff tears.

\section{CONCLUSION}

Handgrip strength did not correlate with pain, range of motion and muscle strength after rotator cuff repair at fourth month follow up. Although an association between grip strength and shoulder function was seen, the correlation was moderate, indicating that multiple factors could contribute to functional outcomes in individuals undergoing rotator cuff repair.

\section{Declaration of conflicting interests}

The author(s) declare no potential conflicts of interest concerning the research, authorship, and/or publication of this article.

\section{REFERENCES}

1. Rahman H, Currier E, Johnson M, Goding R, Johnson AW, Kersh M.E. Primary and Secondary Consequences of Rotator Cuff Injury on Joint Stabilizing Tissues in the Shoulder. J Biomech Eng 2017; 29;139(11):110801-110810.

2. Singh S, Gill S, Mohammad F, Kumar S, Kumar D, Kumar S. Prevalence of shoulder disorders in a tertiary care center. Int J Res Med Sci 2015; 3(4):917-920.

3. Sambandam SN, Khanna V, Gul A, Mounasamy V. Rotator cuff tears: An evidence-based approach. World J Orthop 2015; 6(11):902-18.

4. Oliva F, Piccirilli E, Bossa M, Via AG, Colombo A, Chillemi C et al. I.S.Mu.L.T - Rotator Cuff Tears Guidelines. Muscles Ligaments Tendons J 2016; 5(4):227-263.

5. Pandey V, Willems WJ. Rotator cuff tear : A detailed update. Asia-Pacific J Sport Med Arthrosc Rehabil Technol 2015; 2(1):1-14.

6. Huisstede BMA, Koes BW, Gebremariam L, Keijsers E, Verhaar JA. Current evidence for effectiveness of interventions to treat rotator cuff tears. Man Ther 2011; 16(3):217-30.

7. Yamada N, Hamada K, Nakajima T. Comparison of Conservative and Operative Treatments of Massive Rotator Cuff Tears 2000; 25(4):151-163.

8. Fermont AJ, Wolterbeek N, Wessel RN, Baeyens JP, de Bie R. Prognostic Factors for Successful Recovery After Arthroscopic Rotator Cuff Repair: A Systematic Literature Review. J Orthop Sport Phys Ther 2014; 44(3):153-163.

9. Chen $\mathrm{CH}$, Chang $\mathrm{H}$, Huang YZ, Hung TT. Hand-grip strength is a simple and effective outcome predictor in esophageal cancer following esophagectomy with reconstruction: a prospective study. J Cardiothorac Surg 2011; 6(1):98-102.

10. Sultan P, Hamilton MA, Ackland GL. Preoperative muscle weakness as defined by handgrip strength and postoperative outcomes: a systematic review. BMC Anesthesiol 2012; 12:1.

11. Horsley I, Herrington L, Hoyle R, Prescott E, Bellamy N. Do changes in handgrip strength correlate with shoulder rotator cuff function? Shoulder Elbow 2016; 8(2):124-129.
12. Mandalidis D, O'Brien M. Relationship between hand-grip isometric strength and isokinetic moment data of the shoulder stabilizers. J Bodyw Mov Ther 2010;14(1):19-26.

13. Sporrong H, Palmerud G, Herberts P. Hand grip increases shoulder muscle activity, An EMG analysis with static hand contractions in 9 participants. Acta Orthop Scand 1996; 67(5):485-490.

14. Alizadehkhaiyat O, Fisher AC, Kemp GJ, Vishwanathan K, Frostick SP. Shoulder muscle activation and fatigue during a controlled forceful hand grip task. J Electromyogr Kinesiol 2011; 21(3):478-482.

15. Yun T, Lee B. Effects of hand grip strength on shoulder muscle activity in breast cancer patients. Phys Ther Rehabil Sci 2016;5:95-100.

16. Nascimento LR, Sc M, Polese JC, Sc M, Faria CDCM, et al. Isometric hand grip strength correlated with isokinetic data of the shoulder stabilizers in individuals with chronic stroke. J Bodyw Mov Ther 2012; 16(3):275-280.

17. Budoff JE. The prevalence of rotator cuff weakness in patients with injured hands. J Hand Surg Am 2004; 29(6):1154-1159.

18. Huijing PA, Baan GC. Myofascial force transmission: muscle relative position and length determine agonist and synergist muscle force. J Appl Physiol 2003; 94(3):1092-1107.

19. Walankar P, Verma C, Mehta A. Study of Hand Grip Strength in Indian Population. IJHSR 2016; 6(11):162-166.

20. Yang J, Lin J. Reliability of function-related tests in patients with shoulder pathologies. J Orthop Sports Phys Ther 2006; 36(8):572-576.

21. Norkin CC, White DJ. Measurement Of Joint Motion: A Guide To Goniometry. 4th ed. F.A. Davis; 2009.

22. Jensen MP, Karoly P, Braver S. The measurement of clinical pain intensity: a comparison of six methods. Pain 1986; 27:117-26.

23. Fermont AJ, Wolterbeek N, Wessel RN, Baeyens JP, de Bie RA. Prognostic factors for recovery after arthroscopic rotator cuff repair: a prognostic study. J Shoulder Elbow Surg 2015; 24(8):1249-1256.

24. Damien B, Anshuman S, Sameer S, John GL, Samuel R. Ward, P. The Role of the Peripheral and Central Nervous Systems in Rotator Cuff Disease. J Shoulder Elbow Surg 2015; 24(8):1322-1335.

25. Boileau P, Brassart N, Watkinson DJ, Carles M, Hatzidakis AM, Krishnan SG. Arthroscopic Repair of Full-Thickness Tears of the Supraspinatus: Does the Tendon Really Heal? J Bone Joint Surg Am 2005; 87(6):1229-1240.

26. Chung SW, Kim JY, Kim MH, Kim SH, Oh JH. Arthroscopic Repair of Massive Rotator Cuff Tears: Outcome and Analysis of Factors Associated With Healing Failure or Poor Postoperative Function. Am J Sports Med 2013 30; 41(7):1674-1683.

27. Shin SJ, Chung J, Lee J, Ko YW. Recovery of Muscle Strength After Intact Arthroscopic Rotator Cuff Repair According to Preoperative Rotator Cuff Tear Size. Am J Sports Med 2016; 44(4):972-980.

28. Lefevre-Colau MM, Nguyen C, Palazzo C. et al. Kinematic patterns in normal and degenerative shoulders. Part II: Review of 3-D scapular kinematic patterns in patients with shoulder pain, and clinical implications. Ann Phys Rehabil Med 2018; 61(1):46-53.

29. Leong HT, Ng GY, Chan SC, Fu SN. Rotator cuff tendinopathy alters the muscle activity onset and kinematics of scapula. J Electromyogr Kinesiol 2017; 35:40-46. 